\title{
Acceptation du projet TARCO: redémarrage de la révision tarifaire
}

\section{Urs Stoffel}

Dr méd., membre du Comité central de la FMH, responsable du département Médecine et tarifs ambulatoires

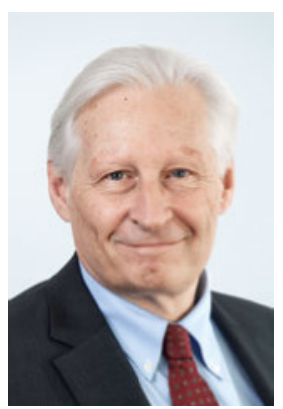

Suite au rejet de la votation générale de juin 2016, les organisations médicales représentées par la FMH ont confirmé que la participation de la FMH au remaniement du TARMED restait absolument nécessaire. Après une analyse détaillée des causes de ce rejet, un projet de révision du tarif médical ambulatoire bénéficiant d'un large soutien a été lancé sous le nom de «TARCO». Après avoir clairement approuvé le plan général TARCO à l'automne 2016, l'Assemblée des délégués de la FMH (AD) a désormais validé à l'unanimité le concept détaillé du projet de révision tarifaire lors de sa séance du 26 janvier 2017, ouvrant ainsi la voie à la mise en œuvre concrète du projet avec les sociétés de discipline médicale et les organisations faîtières.

\section{Objectifs du projet de révision TARCO}

Le projet de révision TARCO a pour objectif d'élaborer d'ici juin 2018 une structure tarifaire revue et adaptée, susceptible d'être acceptée par la majorité du corps médical et de répondre aux exigences légales concernant le caractère approprié et conforme aux règles applicables en économie d'entreprise. Des règles tarifaires obligatoires pour tous et une organisation claire constituent les éléments centraux du projet TARCO. La FMH souhaite terminer les travaux internes d'ici fin 2017. Ensuite, elle entamera les négociations avec les partenaires tarifaires dans le but de déposer en juin 2018 une proposition de tarif, commune et développée en partenariat, pour approbation par le Conseil fédéral. S'agissant des points fondamentaux qui concernent tous les chapitres du tarif, nous les traiterons en partie déjà avec les partenaires tarifaires au cours de la révision interne.

\section{Conditions remplies pour lancer le concept détaillé}

En plus du concept détaillé TARCO, l'AD a aussi approuvé le modèle proposé par le groupe de travail sur les valeurs intrinsèques réglant deux questions fondamentales: la prise en compte des valeurs intrinsèques quantitatives et la définition des critères d'application des valeurs intrinsèques qualitatives. C'était un préalable dans le plan général TARCO pour pouvoir entamer les travaux du concept détaillé.

\section{Facteurs de réussite pour la mise en œuvre du concept détaillé TARCO}

Comme la FMH l'a toujours souligné, la révision tarifaire ne pourra aboutir et être acceptée de tous que si toutes les disciplines spécialisées et toutes les sociétés de discipline médicale impliquées y sont associées. Cela suppose une communication ouverte, régulière et transparente, y compris de la part de la FMH, et en particulier des organisations faîtières et des sociétés de discipline, envers la base. Pour que le projet bénéficie d'une large adhésion, la base doit aussi être entendue. A cet effet, la FMH a mis sur pied des "cafés tarifaires", une plateforme d'échange informelle qui permet aux membres de s'informer de l'avancement du projet de révision et d'y participer activement.

Pour que la révision soit un succès, il faudra cependant dans tous les cas que chacun soit ouvert au compromis, aussi bien les représentants des sociétés à l'interne de la FMH que les partenaires tarifaires.

\section{Garantir l'autonomie tarifaire}

La FMH est convaincue qu'une révision tarifaire globale conforme à la loi et développée en concertation avec tous les partenaires tarifaires est la meilleure voie à suivre. Les interventions ponctuelles dans la structure tarifaire actuelle, telles qu'imposées en octobre 2014 et prévues à partir du $1^{\text {er }}$ janvier 2018 par le Conseil fédéral, ne feront que provoquer des distorsions supplémentaires dans la structure tarifaire TARMED actuellement en vigueur. 